\title{
Decision Support System for Detection of Diabetic Retinopathy Using Smartphones
}

\author{
Prateek Prasanna $^{\star} \quad$ Shubham Jain $^{\ddagger} \quad$ Neelakshi Bhagat ${ }^{\dagger} \quad$ Anant Madabhushi $^{\mp}$ \\ * Department of Electrical and Computer Engineering, Rutgers University, USA \\ $\ddagger$ WINLAB, Rutgers University, USA \\ $\dagger$ Institute of Ophthalmology and Visual Science, UMDNJ, USA \\ $\mp$ Department Of Biomedical Engineering, Case Western Reserve University, USA
}

Email: prateekp@eden.rutgers.edu,shubhamj@winlab.rutgers.edu,bhagatne@umdnj.edu, anant.madabhushi@case.edu

\begin{abstract}
Certain retinal disorders, if not detected in time, can be serious enough to cause blindness in patients. This paper proposes a low-cost and portable smartphone-based decision support system for initial screening of diabetic retinopathy using sophisticated image analysis and machine learning techniques. It requires a smartphone to be attached to a direct hand-held ophthalmoscope. The phone is used to capture fundus images as seen through the direct ophthalmoscope. We deploy pattern recognition on the captured images to develop a classifier that distinguishes normal images from those with retinal abnormalities. The algorithm performance is characterized by testing on an existing database. We were able to diagnose conditions with an average sensitivity of $86 \%$.

Our system has been designed to be used by ophthalmologists, general practitioners, emergency room physicians, and other health care personnel alike. The emphasis of this paper is not only on devising a detection algorithm for diabetic retinopathy, but more so on the development and utility of a novel system for diagnosis. Through this mobile eye-examination system, we envision making the early screening of diabetic retinopathy accessible, especially to rural regions in developing countries, where dedicated ophthalmology centers are expensive, and to alleviate detection in early stages.
\end{abstract}

Keywords-Retinal Diseases, Image Processing, Mobile System, Pattern Recognition, Diabetic Retinopathy

\section{INTRODUCTION}

Diabetic Retinopathy (DR) is in World Health Organization's (WHO) priority list of eye diseases, and one of the leading causes of blindness worldwide [1] [2]. In this paper, we discuss the system developed for the automated detection of $\mathrm{DR}$, which is a complication of diabetes mellitus in the retina, the innermost light-sensitive membrane covering the inside of the eye [2]. It is a result of damaged blood vessels due to long-standing uncontrolled diabetes in the retina.

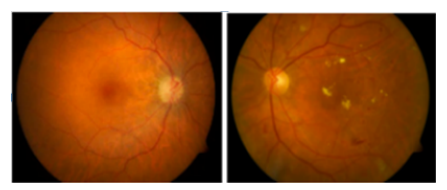

Fig. 1. Fundus images showing normal (left) and abnormal (right) fundus [3].

In DR, blood vessels leak fluid and the retina swells up, and in advanced cases, abnormal new blood vessels (neovascularization) grow on the retinal surface. Microaneurysms, hard exudates, soft exudates, hemorrhages are early changes whereas retinal neovascularization with vitreous hemorrhage are late changes and need emergency treatment to save as much vision as possible.

\section{A. Motivation}

According to WHO, diabetes mellitus currently affects more than 347 million persons worldwide, with the most rapid growth in low and middle-income countries [4]. More than $75 \%$ of patients who have had diabetes for more than 20 years will have some form of DR. WHO has estimated that DR is responsible for $4.8 \%$ of the 37 million cases of blindness throughout the world [5]. In developed countries, the rate of increase is projected at approximately $42 \%$, and in developing countries, particularly in India, it is projected at $150 \%$ [6]. It has been estimated that diabetic individuals are 25 times more likely than their nondiabetic counterparts to suffer severe, permanent vision loss [4]. Recognition of DR in its early asymptomatic stages and early intervention with treatment is the most effective way of preventing permanent vision loss, and can save millions from blindness [7].

The existing solutions to the problem of early detection of DR provide only a detection algorithm that is retrospective in nature, and have not been implemented for practical use. None of the prior works [8] [9] propose an entirely automated and handy system implementation on a mobile device.

\section{B. Background}

Despite the alarming rise of DR all over the world, the performance of evaluation systems, even in developed countries, leaves much to be desired. The existing infrastructure for screening of DR includes ophthalmoscopy, or stereoscopic color film fundus photography. Traditionally, ophthalmologists have performed retinal screening [10]. With advanced technological systems, telemedicine is playing an important role in capturing fundus images, and transmitting photos to ophthalmologists based elsewhere for analysis and assessment. This trend has led to the widespread acquisition and deployment of expensive fundus imaging systems. Experts are being hired to interpret and grade these images. Additionally, there is no universally accepted standard for analysis and classification of retinal images. The final result still depends on the manual expertise of the examiner. With the exponential growth of this problem, introduction of low-cost and timeeffective techniques are a global necessity. On the lines of handy sphygmomanometers, we develop an automated system for detection of DR cases.

Our system offers the following advantages over most conventional equipment and practices:

- Cost-efficiency: Existing methods include costs incurred for expensive sophisticated fundus cameras and operating technicians. Our system, on the other hand, 
uses a low-cost direct ophthalmoscope and smartphone.

- Portability: Our compact system is easy-to-deploy on field locations that are hundreds of miles away from specialists. This is unlike present-day heavy equipment.

- $\quad$ Ease of operation: Presently, ophthalmologists rely extensively on specially trained personnel to capture images using the fundus cameras. Our system simplifies the task of image collection independent of the operator.

- Decision-making capability: In contrast to other systems which require interpretation of images by an ophthalmologist, our system provides a first-hand assessment of conditions to general practitioners and emergency room physicians.

\section{SySTEM DESIGN AND IMPLEMENTATION}

To keep in line with the digital health revolution, and to ensure that health care systems are truly pervasive, we aim at a simple setup that meets the demand for rapidly increasing health services. Fig. 2 provides a representation of our setup, featuring a smartphone and the optical attachment. In the following subsections, we present the system overview, and then elaborate on the detection algorithm and implementation details.

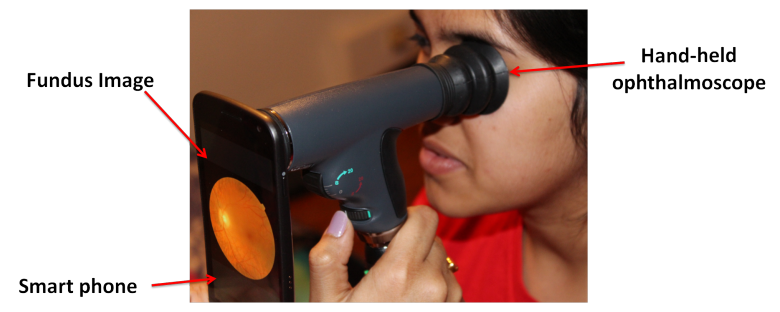

Fig. 2. Our proposed system for fundus imaging.

\section{A. System architecture}

Our system consists of two core hardware parts, (1) a smartphone and (2) a direct hand-held ophthalmoscope. We deploy a smartphone, equipped with the Android mobile operating system. Android is a powerful open-sourced development platform for mobile applications, provided by Google. For carrying out the computer vision operations on the mobile device, we use Intel's OpenCV (Open Source Computer Vision) library [11] which aids real-time image processing. Android's Native Development Kit (NDK) provides the support for compiling and packaging codes written in native-code language, such as $\mathrm{C}++$. The use of native code is ideal for image processing implementation on an Android device, as it provides a build system for efficient and fast processing of CPU-intensive operations. The minimum requirement in terms of smartphone hardware is a high-resolution camera, which most latest generation mobile phones are equipped with.

In our system, we use Google Galaxy Nexus with Android 4.2, JellyBean, the latest version of the Android mobile OS. The smartphone is aligned and attached to a hand-held PanOptic ophthalmoscope [12] which offers a $25^{\circ}$ field of view for a panoramic view of the fundus. We get a $26 \%$ increase in magnification over other standard scopes. These images can be stored on the device for later use. The user interface is designed to be easy for use and intuitive, because the system is intended for use by general medical staff.

\section{B. Detection Algorithm}

Detection of DR involves extraction of blood vessels, microaneurysms and exudates before constructing feature vectors for classification.

1) Detection of blood vessels: The green component of the fundus image is subject to histogram equalization (Fig. 3(b)) followed by morphological opening operation (Fig. 3(c)). This erosion and dilation operation results in diminishing the size of the blood vessels and increasing that of the regions which are not of interest. The outputs of the preceding two steps are subtracted to yield an image that has the optical disk removed (Fig. 3(d)). It is then thresholded to obtain the binary image (Fig. 3(e)). This image contains noise in the form of pixels of small area, that are then filtered to obtain an image with the blood vessels only. The green component of the fundus image is subject to adaptive histogram equalization where the contrast of the image is enhanced by breaking it into sub-blocks (Fig. 3(f)).

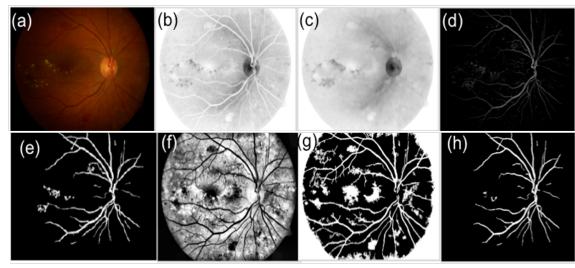

Fig. 3. Various stages of blood vessel extraction.

The output is segmented and then noise is removed (Fig. $3(\mathrm{~g})$ ). This image is further combined with a circular mask at the optic disk region. Creation of the mask involves scanning of the grayscale image to detect the brightest point, which is the center of the optic region. If there are brighter spots, the median is taken. The final output is as shown in Fig. 3(h).

2) Detection of microaneurysms: Microaneurysms are focal dilations of retinal capillaries. Presence of such lesions in the fundus images is a clear sign of DR. The green component (Fig. 5(b)) of the original image (Fig. 5(a)) is extracted and adaptive histogram equalization is applied twice in succession (Fig. 5(c) and Fig. 5(d)). A Canny edge operator is applied on the brightened grayscale image. The eroded version of this image is then subtracted from the dilated version to locate the circular border. Edges of features are obtained by applying canny edge detector on the first adaptive histogram equalized image. This is then subtracted from the border image to obtain an image without the border (Fig. 5(e)). We then fill the holes in the binary edge image and subtract the edge image from the filled image to get Fig. 5(f). Segments in Fig. 5(f) where the total pixels lie below a threshold are removed, and the resulting image is subtracted from Fig. 5(f) to give us microaneurysms with noise (Fig. 5(g)). The second adaptive histogram equalized image is segmented and logically ANDed with Fig. 5(g) to give us an image where exudates have been removed (Fig. 5(h)). The second adaptive histogram equalized image is segmented with a different threshold and its complement gives us an image with blood vessels and noise (Fig. 5(i)). Fig. 5(i) logically ANDed with Fig. 5(g) gives us micro aneurysms after removing blood vessels and noise. After removing the optic disk region, we obtain the final image (Fig. $5(\mathrm{j})$ ) which contains only the microaneurysms. 


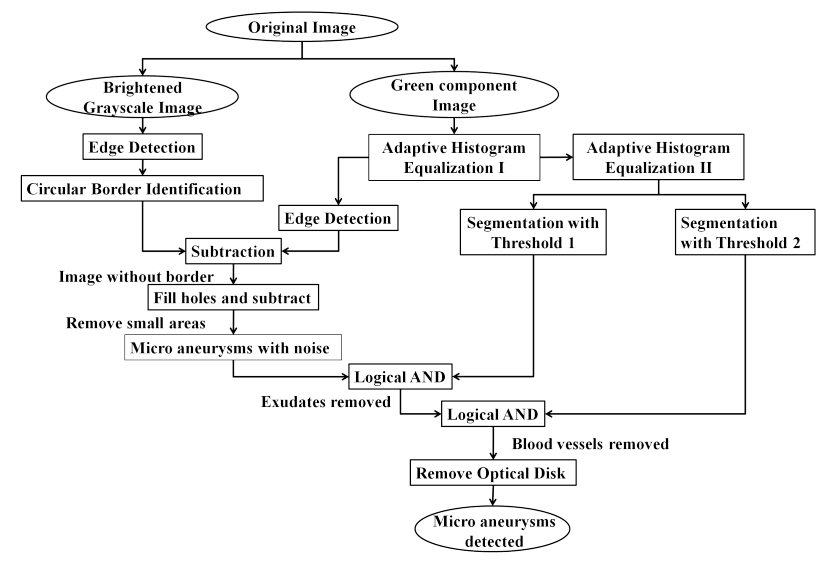

Fig. 4. Step-by-step procedure for detection of microaneurysms.

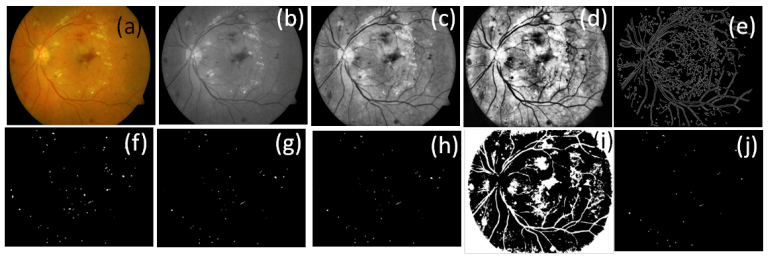

Fig. 5. Intermediate stages in the detection of microaneurysms.

3) Detection of exudates: Exudates are either occlusion of retinal pre-capillary arterioles supplying the nerve fiber layer with concomitant swelling of local nerve fibre axons (soft exudates) or yellow deposits of lipid and protein within the sensory retina (hard exudates). First the grayscale image is brightened (Fig. 7(b)). Edges are then extracted using a canny edge detector (Fig. 7(c)). The holes in Fig. 7(c) are filled, after which its eroded version is subtracted from the dilated version to find the circular border. Morphological closing of Fig. 7(b) yields an image where the blood vessels have been removed (Fig. 7(d)). A column filter is then applied. The resultant image is segmented and the optical disk is removed (Fig. 7(e)). Subtraction of the circular border from Fig. 7(e) yields Fig. 7(f). An adaptive histogram equalization is performed on Fig. 7(b), and the complement of its segmented output gives Fig. 7(g). Morphological closing is applied on Fig. 7(f) and it is logically ANDed with Fig. 7(g) to give the exudates (Fig. 7(h)).

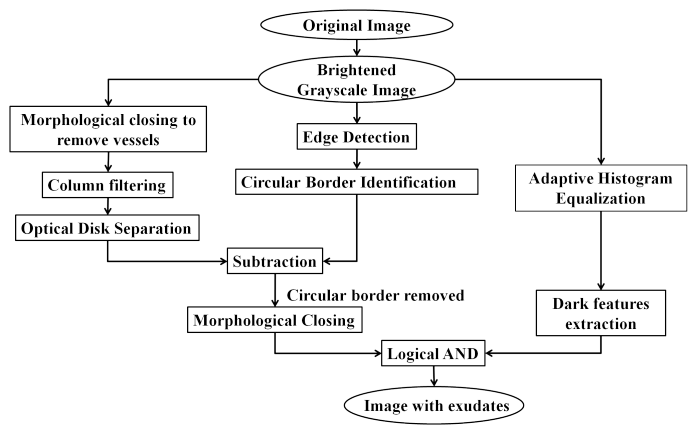

Fig. 6. Step-by-step procedure for detection of exudates.

\section{Implementation}

Before testing on real world data, we analyzed the working of the algorithm on existing database of fundus images. This helped us revisit and strengthen our algorithm. We studied the fundus images, processed them and explored

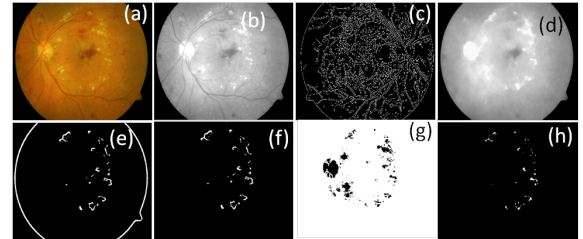

Fig. 7. Intermediate stages in the detection of exudates.

the use of pattern classification techniques to segregate images that have signs of DR from the ones that do not. This would enable ophthalmologists to focus their attention only on images that have disease signs and decide the course of action.

The next step would be finding suitable hardware. For this, we choose a hand-held ophthalmoscope. The ophthalmoscope axis is aligned to the central axis of the phone camera, to capture a precise image of the patient's retina as seen through it. The post processing operations on the fundus images are performed in real-time on the mobile device, and a decision is offered based on the retina's condition. We are currently integrating our algorithm to the mobile application. We are collaborating with ophthalmologists on many fronts, to help us evaluate the requirements of doctors and patients alike, and guide us in making informed choices to make this process more streamlined. This system can prove to be useful for physicians, who can detect diabetes, but do not have the means to check if symptoms for DR exist.

\section{Design Goals and Challenges}

The system has been designed such that each mobile unit is self-contained, being capable of capturing and efficiently processing images on the smartphone with the available resources to provide reliable results. When the system is widely deployed, as a result of which our database will expand, we would be inclined to move our training set to a remote server or a cloud. A large training set, based on the images captured at all locations and various clinical trials, will help us reach improved and more conclusive results. Each autonomous unit will capture an image and process it internally for feature extraction. The feature vector thus obtained can be sent to the cloud, to make use of the updated classifier. All images are stored on a database in the cloud. Such a distributed system benefits from classifier updates in real-time, and provides a reliable detection system. Processing on the cloud facilitates faster implementation and parallel diagnosis of more than one condition. Despite the implementation of a distributed system, each unit would still be independent. In case of a network outage, the detection results can be acquired from a local classifier residing on the mobile device. The local classifier gets updated/synced with the one residing on the cloud when network is available.

The goals will, however, lead to several technical challenges:

- Image quality: Though the system is designed to work on any smartphone, the outcomes of the algorithm rely highly on the quality of the image captured.

- Computation on smartphone: The computation complexity on a smart phone is bounded by available hardware.

- Variation in fundus pigmentation: The system should be tolerant towards changes in fundus pigmentation when tested on individuals from diverse populations. 


\section{PERFORMANCE EVALUATION}

Our initial experiments aimed at developing and testing a detection algorithm on existing databases. Here we evaluate the performance of the algorithm that was designed to detect DR.

\section{A. Dataset}

Four sets of images from the MESSIDOR database [13] were used. These 4 sets comprised 400 images in total with 230 images which exhibited DR condition. This database consisted of images taken with both dilated and undilated pupils.

\section{B. Evaluation}

We construct a $5 \times 1$ feature vector consisting of the area of the blood vessels, areas of lesions (exudates and microaneurysms), the entropy of the image and difference of Gaussian (DoG) values (mean in a region). Our classifier is trained on two-thirds of the data set and validated on the remaining onethird. We used support vector machine (SVM), Adaboost.M1 and Logistic regression classifiers. Logistic regression was found to be the best performing classifier with an area under curve (AUC) of 0.844 . The receiver operating characteristic (ROC) curve is shown in Fig. 8. The average sensitivity of the system was found to be $86 \%[14]$. We intend to improve the sensitivity by training our classifier on large and varied databases. Also, we would explore the use of more robust features $[15]$ for improving upon the detection rate of blood vessels.

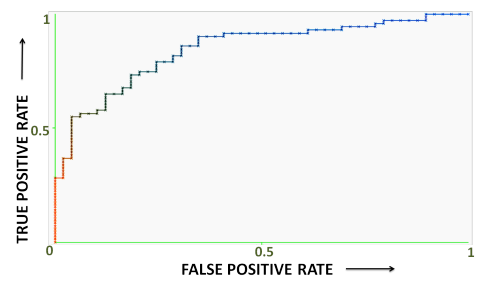

Fig. 8. The receiver operating characteristic (ROC) curve for the decision system.

\section{Social Implication}

Our cost-effective solution seeks to provide appropriate and feasible eye-care, especially targeted to rural regions in developing countries, where dedicated ophthalmology centers are expensive. Such a system could easily be incorporated in mobile health-care stations and health camps set up at different locations. Patients who find it difficult to sit upright can have their eyes examined, even when lying down.

In the long run, the utility of our system would be manifold. This system would save the human labor and time spent in analyzing normal images with no evidence of diabetic changes and only images with some disease process can be separated and sent to the appropriate personnel to grade. In medical schools, instructors would be able to use this system as a standard for their teaching, and students would get their first good look at the retinal changes. The residents-in-training, or emergency room physicians will be able to capture the abnormal images and send them to ophthalmologists not onsite. It can also be used by veterinarians.

Thus, our intelligent system aims at improving opportunities in health-care across the developing world, and also in the developed countries where health insurance costs are rising. It is part of a bigger revolution, contributing to the future of medicine by providing medical professionals with low-cost hardware and efficient software, the ability to capture fundus images and get an initial assessment of many vision threatening diseases that affect the central $25^{\circ}$ of the retina; this may be DR, macular lesions, retinal detachments, or age-related macular degeneration. This would lead to early detection of such diseases and hopefully early intervention to preserve as much vision as possible, and reduce blindness

\section{CONCLUSION AND FUTURE WORK}

We have presented the design and implementation of a system that utilizes a smartphone as a platform for computer aided diagnosis and screening of DR. This is achieved by attaching a hand-held direct ophthalmoscope to the phone. Our mobile application captures the ophthalmoscope images of the retina, and applies pattern recognition and statistical inference algorithms to facilitate decision making for the initial screening of DR. We discussed our detection algorithm and the performance numbers attained on an existing standard database. The automated system is a stand-alone arrangement that uses a classifier and a large training set for conclusive results. We also proposed the system design for rendering the scalability of the system, thereby decreasing the problems associated with clinician inter-observer variability.

Further, once the system has been trained to recognize basic irregularities in the retina, it can be extrapolated to work for screening other retinal conditions too. This lowcost examination system is ideal for underdeveloped regions to promote quality health care and make it available to one and all. This is potentially a powerful tool for next generation implementation of pervasive healthcare systems.

\section{REFERENCES}

[1] "Blindness," http://www.who.int/blindness/causes/priority/en/index6.html.

[2] "NEI," http://www.nei.nih.gov/health/diabetic/retinopathy.asp.

[3] "DIARETDB0: Standard Diabetic Retinopathy Database," http://www2.it.lut.fi/project/imageret/diaretdb0/index.html.

[4] “WHO," http://www.who.int/diabetes/en/index.html.

[5] "WHO-Blindness-Prevention," http://www.who.int/blindness/Prevention of Blindness from Diabetes Mellitus-with-cover-small.pdf.

[6] "IDOS," http://theidos.com/telescreening-for-diabetic.aspx.

[7] "Early Treatment Diabetic Retinopathy Study (ETDRS)," http://www.nei.nih.gov/neitrials/static/study53.asp.

[8] D. Usher, M. Dumskyj, M. Himaga, T. H. Williamson, S. Nussey, and J. Boyce, "Automated detection of diabetic retinopathy in digital retinal images: a tool for diabetic retinopathy screening," Diabetic Medicine, 2004

[9] T. Walter, J.-C. Klein, P. Massin, and A. Erginay, "A contribution of image processing to the diagnosis of diabetic retinopathy-detection of exudates in color fundus images of the human retina," Medical Imaging, IEEE Transactions on, vol. 21, no. 10, pp. 1236 -1243, oct. 2002.

[10] "Diabetes-journal," http://clinical.diabetesjournals.org/content/27/4/140.full.

[11] G. Bradski, "The OpenCV Library," Dr. Dobb's Journal of Software Tools, 2000.

[12] "PanOptic," www.welchallyn.com/promotions/PanOptic/default.htm.

[13] "MESSIDOR database," http://messidor.crihan.fr/.

[14] Mark Hall, Eibe Frank, Geoffrey Holmes, Bernhard Pfahringer, Peter Reutemann, and Ian H. Witten, "The weka data mining software: An update," SIGKDD, 2009.

[15] Prateek Prasanna, Kristin Dana, Nenad Gucunski, and Basily Basily, "Computer-vision based crack detection and analysis," in SPIE Smart Structures and Materials + Nondestructive Evaluation and Health Monitoring. International Society for Optics and Photonics, 2012, pp. 834542-834542. 